\title{
Geostatical Characterisation of a Carbonate Reservoir Located in South East Turkey
}

\author{
Gümrah F., Akin S., Yeten B. \\ Middle East Technical University, \\ Petr. Eng. Dept., Turkey
}

\begin{abstract}
Copyright 1995, Steering Committee of the European IOR - Symposium.
This paper was presented at the 8th. European IOR - Symposium in Vienna, Austria, May 16 - 17, 1995

This paper was selected for presentation by the Steering Committee, following review of information contained in an abstract

submitted by the author(s). The paper, as presented has not been reviewed by the Steering Committee.
\end{abstract}

\begin{abstract}
Spurred by the enormous expense of developing heterogeneous fields and by the desire of oil companies to increase ultimate recovery, the science of reservoir characterization is making rapid progress. Reservoir characterization encompasses all techniques and methods that improve understanding the geological and petrophysical controls of fluid flow. The objective is to provide practical reservoir models for optimum field development.
\end{abstract}

This paper presents a comprehensive geostatistical evaluation of the distribution of interwell porosity and permeability in a carbonate reservoir located in the south east part of Turkey. Major geological units were firstly identified using well log and core data, and then divided into subzones. A zonation technique was used to identify and describe naturally occurring zones in the reservoir. The variation of geologic and petrophysical properties that exist within each subzone was defined. A kriging technique was used to produce statistical distributions of reservoir properties between wells at the reservoir scale. Finally, an input data file for the simulation of the production performance of the field was developed.

\section{Introduction}

An accurate reservoir description - the internal, three dimensional variation of reservoir rock properties - or geological complexity is essential to effective reservoir management(1). During primary production, areal variation of properties such as permeability, porosity, thickness and saturation influence both oil recovery and its distribution in the field. During secondary and tertiary production, vertical reservoir heterogeneity is a predominant factor affecting oil recovery. Knowledge of reservoir geology is particularly important when reservoir simulation models are used to evaluate and predict reservoir performance.

The problem of defining the geologic complexity can be divided into three stages:

- Defining the reservoir's macro structure using deterministic data.

- Defining the micro scale variations using statistical techniques geostatistics.

- Readjusting the detailed geologic model to be suitable input for a reservoir simulator.

The first stage aims to construct a geologic description using the seismic, 
static data from cores and logs, and dynamic production data from well testing. In this stage lithological or depositional units are identified and if possible subdivided into subzones. Flow units represent an assemblage of facies having similar characteristics. The significance of dividing sedimentary intervals into flow units is that each flow unit usually reflects a specific depositional environment and the characteristics of fluid flow. In most cases, this macro scale characterization results in a layer cake model. However, more complex arrangements such as jigsaw and labyrinth models are reported in the literature (2).

Once the large-scale structure has been defined, the next stage focuses on defining the complexity within each subzone generated in the first stage. Lithofacies correlation and associated petrophysical values such as porosity and permeability are commonly forced from well to well by geostatistical techniques. For a given property, a variogram is constructed from pairs of data generally measured in wells. All of the computed points on the variograms are fit by mathematical models that best captured the vertical and horizontal behaviour using a method called, indicative goodness of fit $(I G F)^{(3)}$. As the name implies it is a measure of how good the fit represents the original variogram.

After modelling is complete the next step is to estimate the values of petrophysical variables at unsampled locations. A geostatistical technique used for estimation purposes in conventional analogy is commonly called kriging ${ }^{(4)}$. At this point, conditional simulation which is a geostatistical method can be used to generate equiprobable reservoir description(5). The final stage consists of scaling up or enshrining the high resolution petrophysical data into smaller number of larger grid blocks, so that the computer storage problem is solved. To preserve the effect of heterogeneity at all scales, a series of scale up operations can be performed, each dealing with heterogeneity larger than the previous operation.

Although it is generally thought that the data from at least 20 or 30 wells should be used for a well description, An Ping Yang(6) states that it is possible to use as few as two wells to generate a crosssection, if a fractal approach is used. In this study, instead of a fractal approach we consider the utilization of conventional characterization techniques combined with geological information to generate a cross-section.

A carbonate reservoir located in the south east part of Turkey was characterized using the methodology described above.

\section{Description Of The Field}

CBS field, located in the vicinity of Siverek in south east Turkey, is explored and developed by Turkish Petroleum Company. The field is a rather small one, consists of 6 wells, two of which is producing right now. The well spacing is about 400 meters and the wells are drilled randomly. At both of the wells the movable hydrocarbon (13 ${ }^{\circ} \mathrm{API}$ crude oil) seems to be in KBB formation as observed from the logs. This formation is a fractured carbonate and the flow is mainly from the fractures which are continuos and hair like. The target formation consists of mainly limestone with traces of shale and dolomite. The company has decided not to perforate the CBS 2 well due to the results of DST and coring operations for which the permeability is thought to be low. However CBS 1 well is perforated to produce oil between the intervals [1489-1507m], [1526-1551m] and [1157-1565m]. The rest of the wells are closed because of economical reasons.

\section{Characterization Of The Field}

Using the methodology described previously CBS field was characterized. Because of the current situation of the field the characterization was done 
between two wells. First the petrophysical values were gathered using the $\log$ and core data and then these were used in combination with the geological data gathered during drilling to generate flow units. It has been observed that there are three main formations within the reservoir : KBBB, KBBC and $K B G Z$ as shown in Figure 1. Although both of these formations are possible oil producing zones only KBBC formation was perforated by the company. Because of this fact it was decided to focus on this zone. However for the sake of completeness the other zones that are present within the reservoir were characterized too.

In order to determine the flow units of KBBC formation, which seems to be the most prospectful formation throughout the field, the log porosity data of this formation was transformed to core porosity data by utilizing the following relation :

$\phi_{\text {core }}=(1.12043945) \cdot \phi_{\text {log }}$

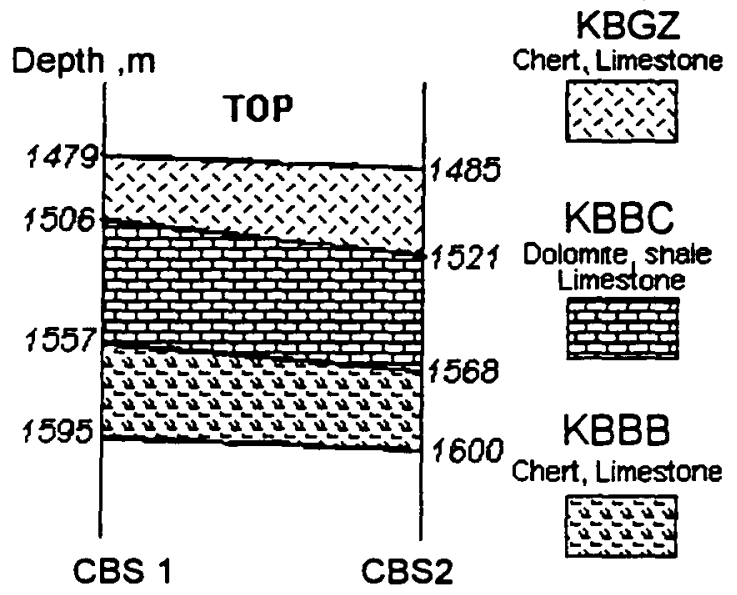

Figure 1-Zonation Diagram of the CBS Field

Then for the same depths by using a logarithmic porosity - permeability relationship transformed core porosity is related to core permeability as shown below:

$\log k=(0.1555) \phi_{\text {core }}-(2.2773)$

Although it has been reported by Lucia(7) that permeability was not directly related to porosity because of the great variability in the pore geometry for carbonates, for the sake of simplicity it has been assumed that the above relationship holds for this zone. Having the above relationship established, permeability data wàs generated for each subsequent depth, and a data file is prepared for each zone. The data file consisted of depth, log porosity and permeability values for each formation. Then these were used to analyze the basic statistical parameters like the distribution type and the histogram of the data via a software, namely GeoEAS (Geostatical Environmental Assessment Software) which is a collection of interactive software tools for performing two-dimensional geostatistical analysis of spatially distributed data( ${ }^{(8)}$. The corresponding plots presented in Figures 2 and 3 were obtained for KBBC zone. It can be easily observed that porosity distributions are normal whereas permeability distributions are log normal.

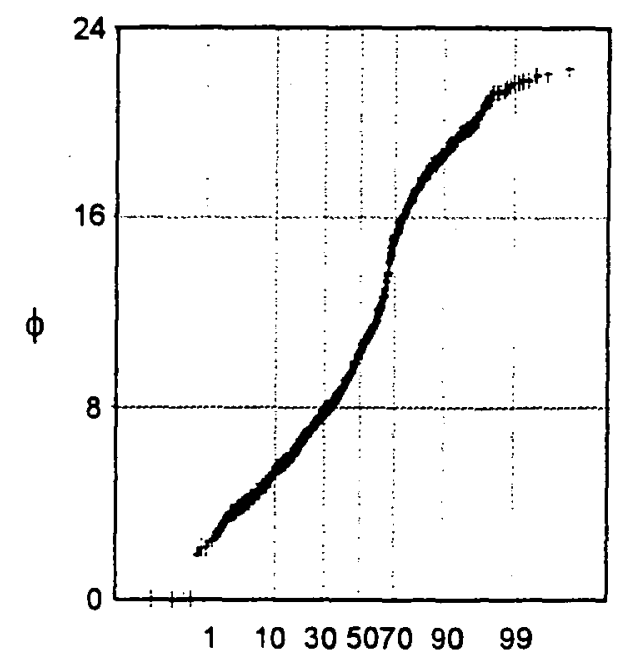

Cumulative $\%$

Figure 2. Normal Distribution of Porosity through KBBC Zone

Then these raw data was fed to another software because of the memory limitations of Geo-EAS, VarioWIN to create pair comparison files (pcf)(3). These files are then used to create variograms. Creating a variogram for a zone needs a tedious work. One should estimate such 
lag distances that the resulting variogram represents similar characteristics by changing the lag distances with little increments or decrements. Then models are fit to these variograms and IGF analysis is performed.

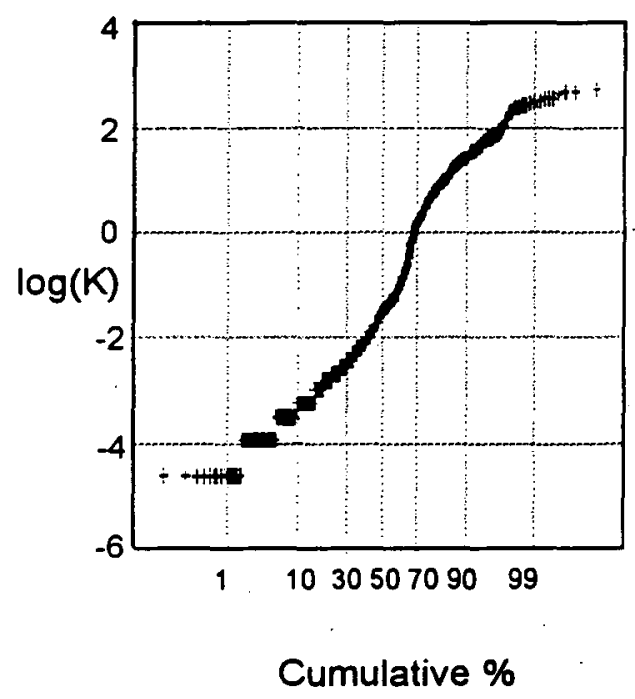

Figure 3. Log Normal Distribution of Permeability through KBBC Zone

The IGF is calculated every time the $2 D$ general model is modified. It gives a measure of how well the model adjusts the directional (cross) variogram. It is mathematically presented by the following equation:

IGF $=\frac{1}{N} \sum_{k=1}^{N} \sum_{i=0}^{n(k)} \frac{h \max (k)}{\sum_{j=0}^{n(k)} P(j)} \cdot \frac{P(i)}{h(i)} \cdot\left[\frac{\gamma(i)-\gamma^{*}(i)}{\sigma^{2}}\right]^{2} \cdot$

The IGF is a number without units and a value close to zero indicates a good fit. Since it is a standardized measure of fit, its value is comparable from one modelling session to another allowing the user to check how well the models fit the experimental measures numerically. Figures 5 through 8 shows the variograms of porosity and permeability generated using the above methodology. It is interesting to note that each variogram has a specific and different model fit which ensures the flow unit determination process carried out previously. In other words, the variograms successfully identified patterns of heterogeneity.

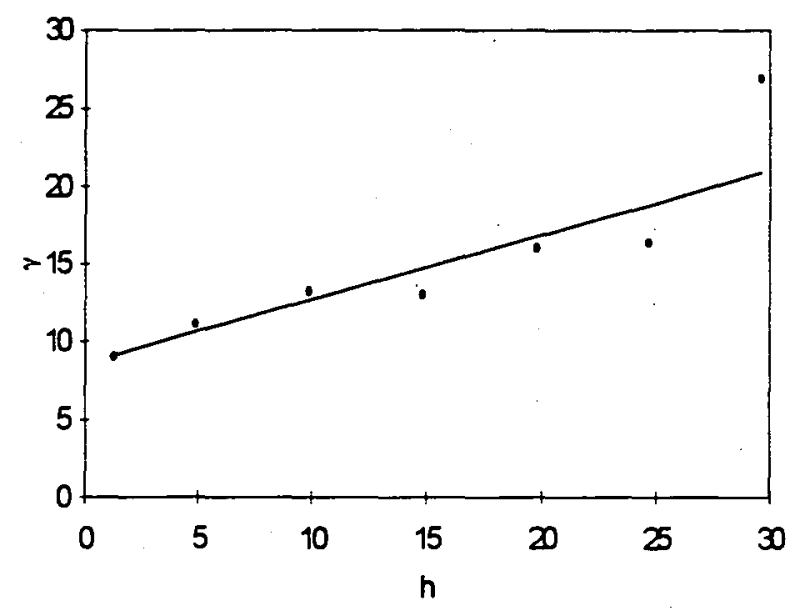

Linear Model

$$
\gamma(\mathrm{h})=8.549+0.41556 \cdot(\mathrm{h})
$$

IGF : $2.3827 .10^{-2}$

Figure 5. Variogram and Model of Porosity fit for KBGZ formation

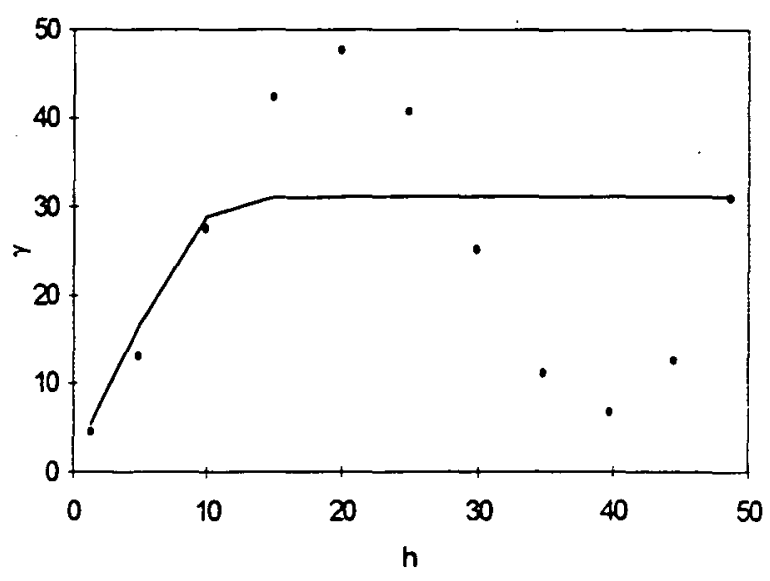

Gaussian Model

$\gamma(h)=4.1931+27.0 \cdot\left[1-\exp \left(\frac{-3 h^{2}}{(10.923)^{2}}\right)\right]$

IGF : $5.10^{-1}$

Figure 6. Variogram and Model fit of Porosity for KBBC Formation

Then via these models the data is block krigged by utilizing Geo-EAS. In order to 
check whether the created variograms are representing the original data, cross validation is performed by using the same software. Cross validation is carried out at the well locations i.e., comparing the estimated data with the known log data. It has been observed that a very well correlation exists with the estimated data and the known data as. The results are presented as contour maps which can be seen in Figures 11 through 14.

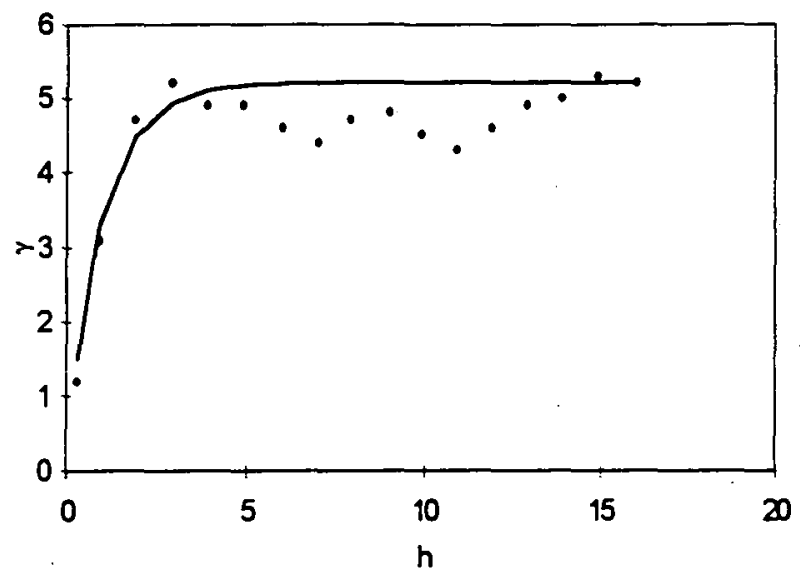

Exponential Model
$\gamma(h)=0.454913+4.75953 \cdot\left[1-\exp \left(\frac{-3 h}{3.1114}\right)\right]$

IGF : 2.075.10-2

Figure 7. Variogram and Model fit of Porosity for KBBB Formation

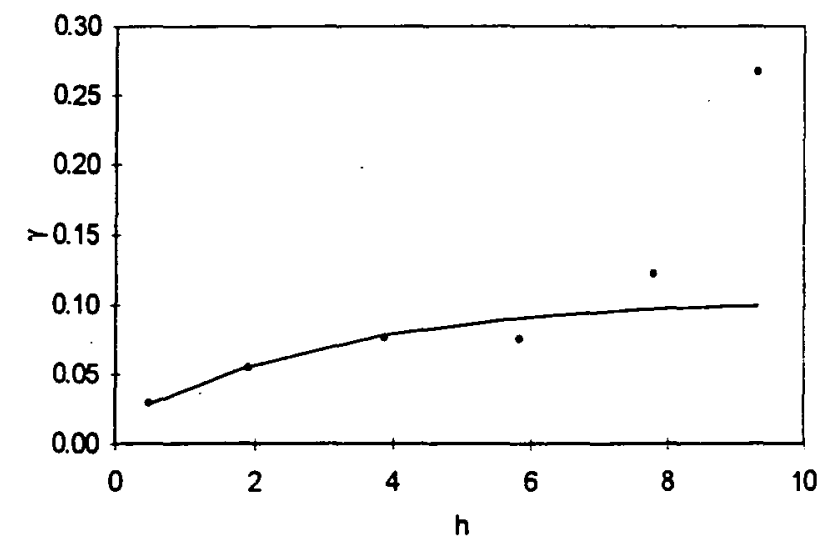

$\gamma(h)=0.015+0.08908 \cdot\left[1-\exp \left(\frac{-3 h}{9.4}\right)\right]$

IGF : $3.6257 .10^{-2}$

Figure 8. Variogram and Model fit of

Permeability for KBBC Formation
After variogram analysis flow units of the formations were identified. The main criterion to establish the flow : units is evaluating the derivative of the permeability with respect to depth. To do this finite difference formulas for equally spaced data were used(9). Since the drilled wells penetrated the KBBC formation at different vertical distances, these derivatives were plotted with respect to depth for each well as shown in Figure 9 and 10. A subzone throughout the KBBC formation is developed via inspecting the derivative of permeability plots. The following zones are correlated : [1531$1536.9 \mathrm{~m}]$ for CBS1 and [1532.1-1542m] for CBS2, and the resulting permeability estimate is obtained using the aforementioned subzones.

The resulting cross-validation plots of the models of porosity and permeability distribution of KBBC formation which is presented by Figures 15 and 16 respectively, indicate that the models perfectly fit to the data which can also be verified by checking out the IGF results of the corresponding fits.

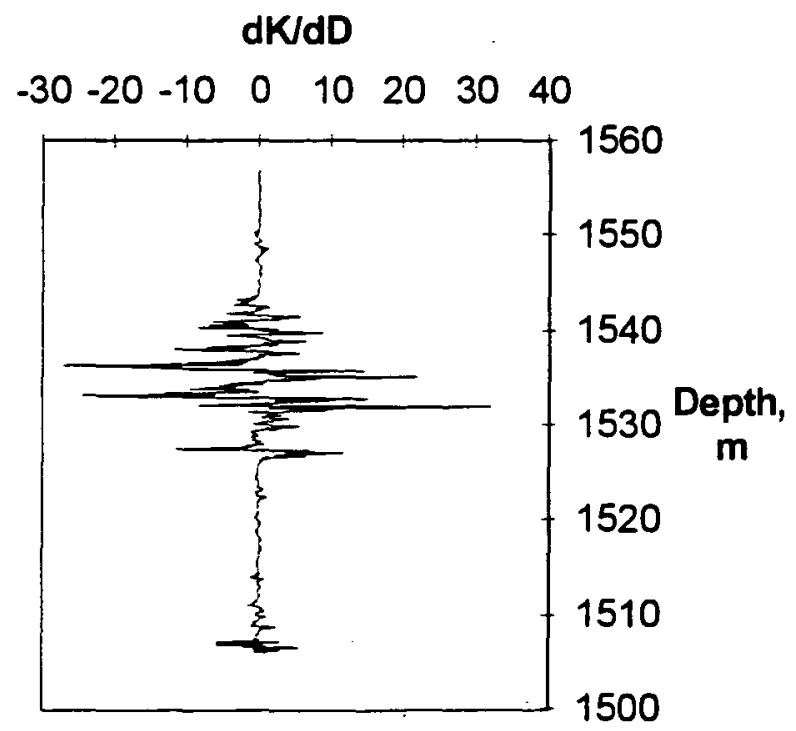

Figure 9. Derivative of Permeability Plot of well CBS1 through KBBC Zone 


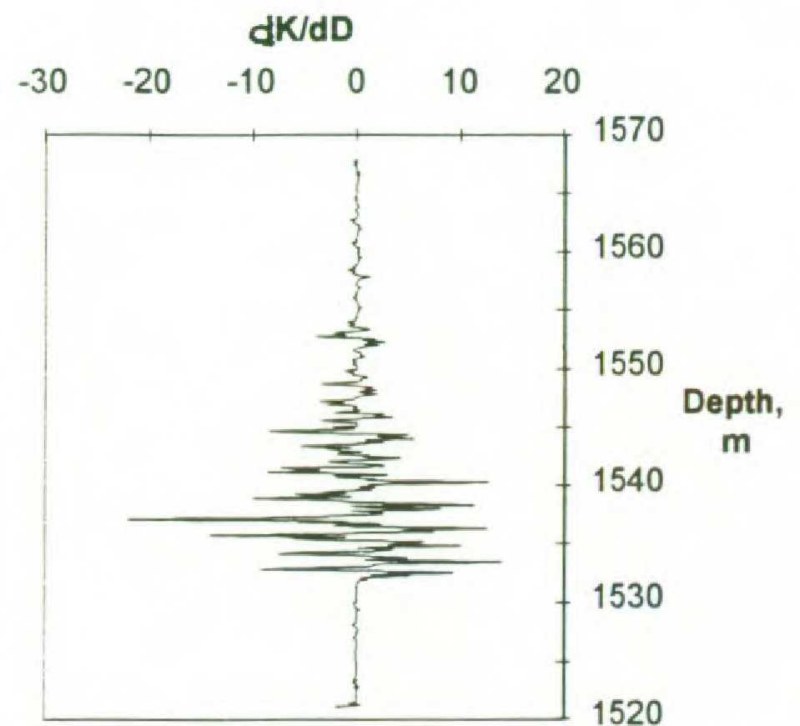

Figure 10. Derivative of Permeability Plot of well CBS2 through $\mathrm{KBBC}$ zone

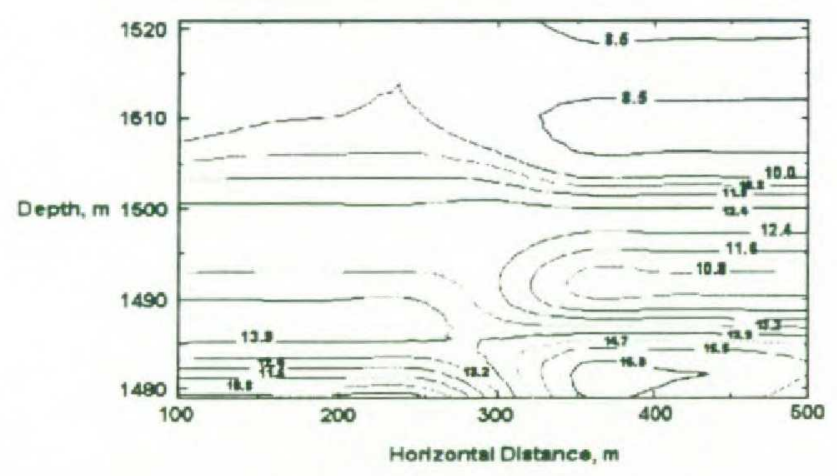

Figure 11. KBGZ Porosity contour Map

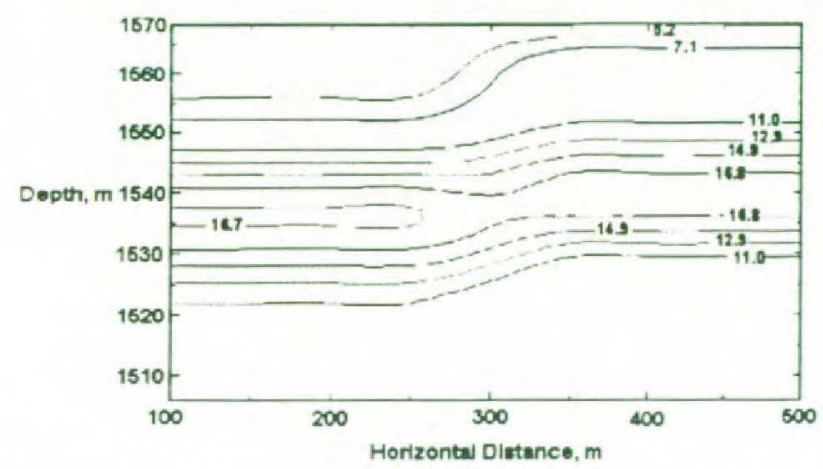

Figure 12. KBBC Porosity Contour Map

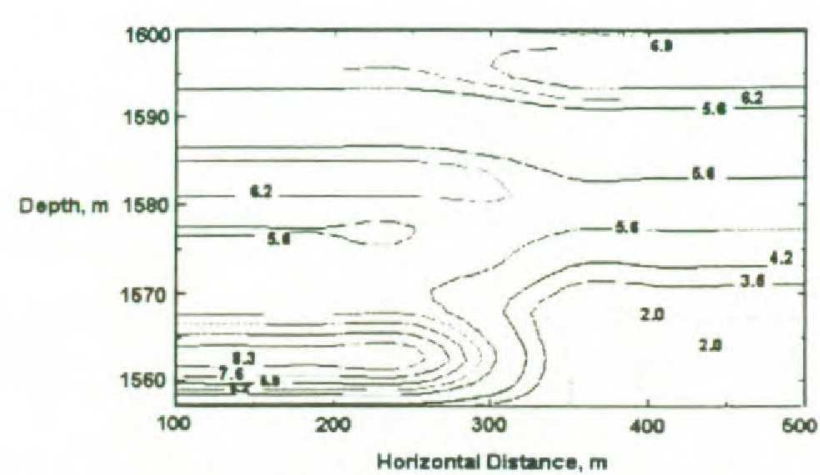

Figure 13. KBBB Porosity Contour Map

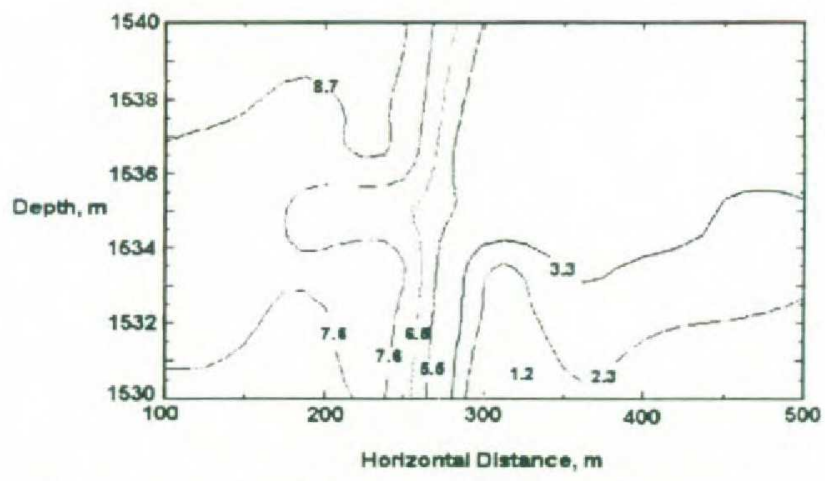

Figure 14. KBBC Permeability Contour Map

Having the characterization completed the next step performed was the preparation of an input file to a simulator. The simulator utilized was the well known $B O A S T(10)$. An attractive feature of the BOAST simulator is that it was developed by DOE, extensively tested, and is publicly available at low cost. Because of the limitations in computer memory the permeability and porosity data generated previously need to be scaled again. In order to achieve this goal pseudo functions that couple rock capillary pressure curves with the petrophysical data need to be defined as reported by Kyte and Berry(11). However it is also possible to repeat the characterization process with a spatial distance larger compared to the original case for scaling purposes. Test cases were tried on a cross-section water flooding scheme utilizing a $25 \times 25,10 \times 10$, and finally $5 \times 5$ resolution. It has been observed that the flow behaviour of the detailed model was 
anisotropic, whereas the simpler approach yielded more isotropic behaviour and sigignificantly higher recoveries as observed from Figure 17. This behaviour was also observed when pseudo functions are used(11). So, it has been concluded that small-scale heterogeneity affects reservoir producibility, and small-scale structure cannot be ignored. However it should be noted that because of the simplifications, it is not possible to relate this cross-section problem directly to the field problem.

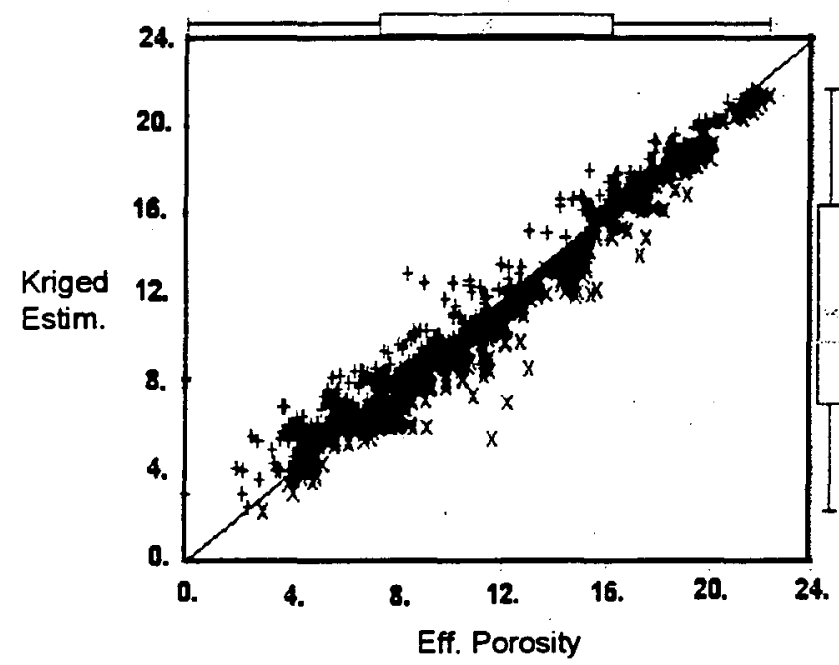

Figure 15. Cross Validation Plot for Porosity Distribution of KBBC Formation

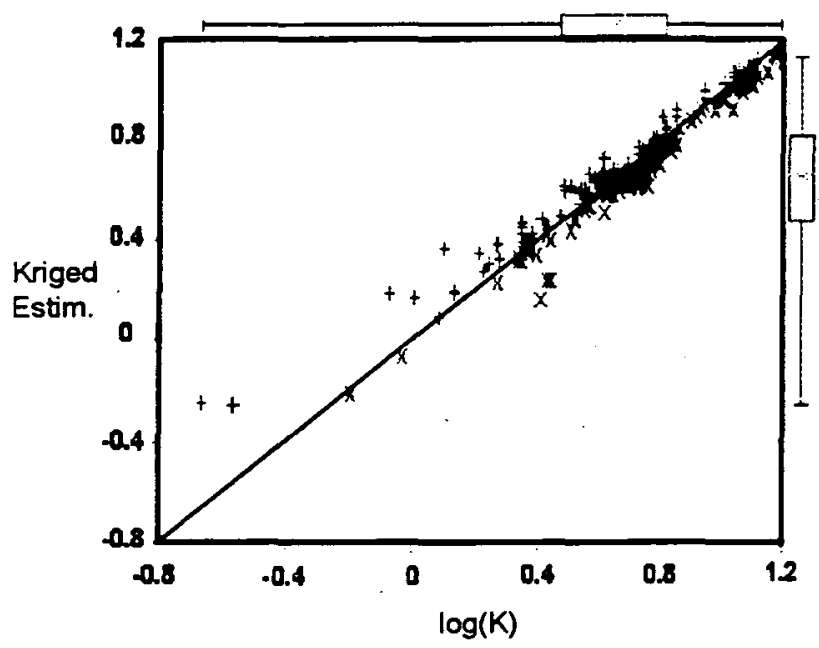

Figure 16. Cross Validation Plot for Permeability Distribution of KBBC Formation

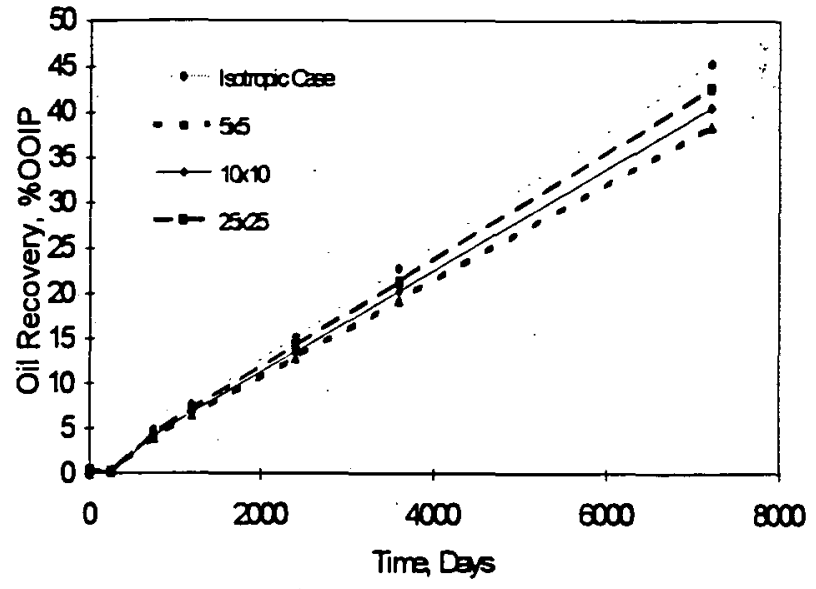

Figure 17. Effect of Scaling Up on Oil Recovery

\section{Conclusions}

A highly heterogeneous carbonate reservoir located in south east Turkey was characterized using combined geostatistical and geological modelling. Based on the results of the study the following conclusions were reached:

1. The reservoir was divided into subzones and analyzed independently. The resulting models which are different from each other showed that the assumption of stationarity was proper for this study.

2. The scarcity of the data let us to characterize the field in two dimensions.

3. A simulator input file was generated using the generated petrophysical data. Kriging techniques were used instead of pseudo functions for scaling purposes. It has been observed that scaling results in loss of heterogeneity.

\section{Acknowledgements}

We would like to thank to the Turkish Petroleum Oil Company (TPOC) for giving us the permission to publish this paper. We also thank many colleagues in TPOC who contributed to this work, particularly Can Bakiler and Yildiz Karakeçe who supplied the necessary data. Yvan Pannatier deserves special thanks for 
helping us getting used to his computer programme, namely VarioWIN.

\section{Nomenclature}

$\phi \quad$ : porosity, fractions

$\mathrm{K}$ : permeability, md

$\mathrm{N}$ : number of directional variogram measures used for the model

$n(k) \quad$ : number of lag for the $k^{\text {th }}$ variogram measure

$P(i)$ : number of pair for lag $i$

h(i) : mean distance for lag i

$h_{\max }(k)$ : maximum distance for the $k$ th variogram measure

$\gamma(i)$ : experimental variogram measure for lag $i$

$\gamma^{*}(i)$ : modeled variogram measure for the mean distance of lag $i$

$\sigma^{2} \quad$ : variance of the data

\section{References}

1. Harris, D.G., and Hewitt, C.H.: "Synergism in Reservoir Management," Journal of Petroleum Technology, July $1977,761-70$

2. Weber, K.J. and van Geuns, L.C. :"Framework For Constructing Clastic Reservoir Simulation Models," Journal of Petroleum Technology, Oct. 1990, 12481253, 1296-1297.

3. Pannatier, $Y$. :MS-WINDOWS Programs For Exploratory Variography and Variogram Modeling in 2D," Statistics of Spatial Processes: Theory and Applications, Capasso V. , Gireone G., and Pos D. (eds), Bari, Italy, Sept. 29-30, 1993, 165-170.

4. Krige, D.G. :"A Statistical Analysis of Some of the Borehole Values of the Orange Free State Goldfield," Journal of Chemical, Metallurgical and Mining Society of South Africa, No 53, 1952, 4770.
5. Journel A.G. and Alabert G.F. :"New Method For Reservoir Mapping," Journal of Petroleum Technology, Feb. 1990, 212218.

6. Yang. A. P., "A Geostatistical Interpolation Method From Only Two or Three Wells", SPE 22346, SPE International Meeting on Petroleum Engineering, Beijing, China, March 24-27, 1991, 177-186.

7. Lucia, F.J. and Fogg, G.E. :"Geologic / Stochastic Mapping Heterogeneity in a Carbonate Reservoir," Journal of Petroleum Technology, Oct. 1990, 12981303.

8. Englund. E. and Sparks. A., "GEO-EAS (Geostatistical Assessment Software) User's Guide", U.S.A., September 1988

9. Kara. B. ,"Quantitative Characterization of Valley-Fill Deposits : Application to Muddy Sandstone in Newcastle, Wyoming", MS Thesis, 1993, The University of Tulsa, OK, U.S.A.

10. Stapp, L.G., and Allison, E.C. :"Handbook For Personal Computer Version of BOAST II : A Three Dimensional, Three-Phase Black Oil Applied Simulation Tool, Department of Energy, Bartlessville, Oklahoma, USA, Dec. 1989.

11. Kyte, J.R., and Berry, D.W. :New Pseudo Functions to Control Numerical Dispersion," Society of Petroleum Engineers Journal, Aug. 1975, 269-276. 